\title{
Engineering of the Magnetized Target Fusion Propulsion System
}

\author{
G. Statham ${ }^{1,2}$, S. White ${ }^{1,2}$, R.B. Adams ${ }^{1}$, Y.C.F. Thio ${ }^{3}$, J. Santarius ${ }^{4}$, \\ R. Alexander ${ }^{1}$, J. Chapman ${ }^{1}$, S. Fincher ${ }^{1}$, A. Philips ${ }^{1}$ and T. Polsgrove ${ }^{1}$ \\ ${ }^{I}$ NASA Marshall Space Flight Center, Advanced Concepts Department, Huntsville, AL 35812, USA. \\ ${ }^{2}$ ERC Inc., 555 Sparkman Drive, Executive Plaza, Suite 1622, Huntsville, AL 35816, USA. \\ ${ }^{3}$ U.S. Department of Energy, Office of Fusion Energy Sciences, 19901 Germantown Road, Germantown, MD \\ 20874, USA. \\ ${ }^{4}$ Institute of Fusion Technology, University of Wisconsin, Madison, WI 20874, USA.
}

\begin{abstract}
Engineering details are presented for a magnetized target fusion (MTF) propulsion system designed to support crewed missions to the outer solar system. Basic operation of an MTF propulsion system is introduced. Structural, thermal, radiation-management and electrical design details are presented. The propellant storage and supply system design is also presented. A propulsion system mass estimate and associated performance figures are given. The advantages of helium- 3 as a fusion fuel for an advanced MTF system are discussed.
\end{abstract}

\section{INTRODUCTION}

Magnetized Target Fusion (MTF) is a propulsion technique that combines features from both inertial and magnetic confinement fusion approaches and capitalizes on research results in both areas (Thio, 1999). The MTF technique offers the promise of both high specific impulse and low dry mass; as such it is well suited to the demands of high delta-v travel to the outer solar system, including human exploration missions. The work reported in this paper was carried out as part of the Human Outer Planet Exploration (HOPE) study, part of the Revolutionary Aerospace Concepts (RASC) program. The HOPE objective was to design a vehicle capable of conducting a human exploration mission of the Jovian moon Callisto. The MTF propulsion system design, as reported here, was developed to satisfy the requirements of this mission. Details of the mission and vehicle are reported separately (Adams, 2003).

This paper is organized as follows. First a brief overview is presented, addressing the basic processes involved in MTF operation. For the purposes of this description, the main fusion fuel is assumed to be deuterium. The subsequent sections are devoted to engineering descriptions of the major MTF components. Finally, the more advanced MTF system, using a mixture of deuterium and helium-3 as the main fusion fuel, is introduced.

\section{BASIC MTF OPERATION}

In outline the MTF system operates as follows. A small plasma target comprised of an easily-ignitable fuel (a $50 \%$ deuterium - 50\% tritium molar mixture) is compressed to fusion conditions by converging streams of high-speed plasma, produced by an array of magnetoplasmadynamic (MPD) accelerators, also known as plasma guns. Energy released from the deuterium-tritium reactions initiates deuterium-deuterium fusion reactions within a deuterium layer immediately surrounding the target. The use of a deuterium-tritium target, with its relatively low ignition temperature, makes the initial fusion burn relatively easy to initiate, but the main energy 
release comes from the deuterium-deuterium reactions in the surrounding layer. This minimizes the need for tritium, which is relatively scarce and expensive. It also minimizes the number of high energy neutrons produced during fusion; those produced by the deuterium-deuterium reaction being of much lower energy than those created from deuterium-tritium fusion (2.45 MeV as opposed to $14.1 \mathrm{MeV}$ ).

The energy release during fusion produces a very rapidly expanding hot plasma. By initiating the fusion event within the confines of a magnetic nozzle, part of the momentum of this expanding plasma cloud can be used to deflect and compress the magnetic field and thus exert a thrust on the vehicle.

The compression and fusion processes are illustrated conceptually in Figures 1 through 4, none of which are to scale.

Figure 1 shows a cross section through the plasma target with the various high-speed plasma streams converging on it.

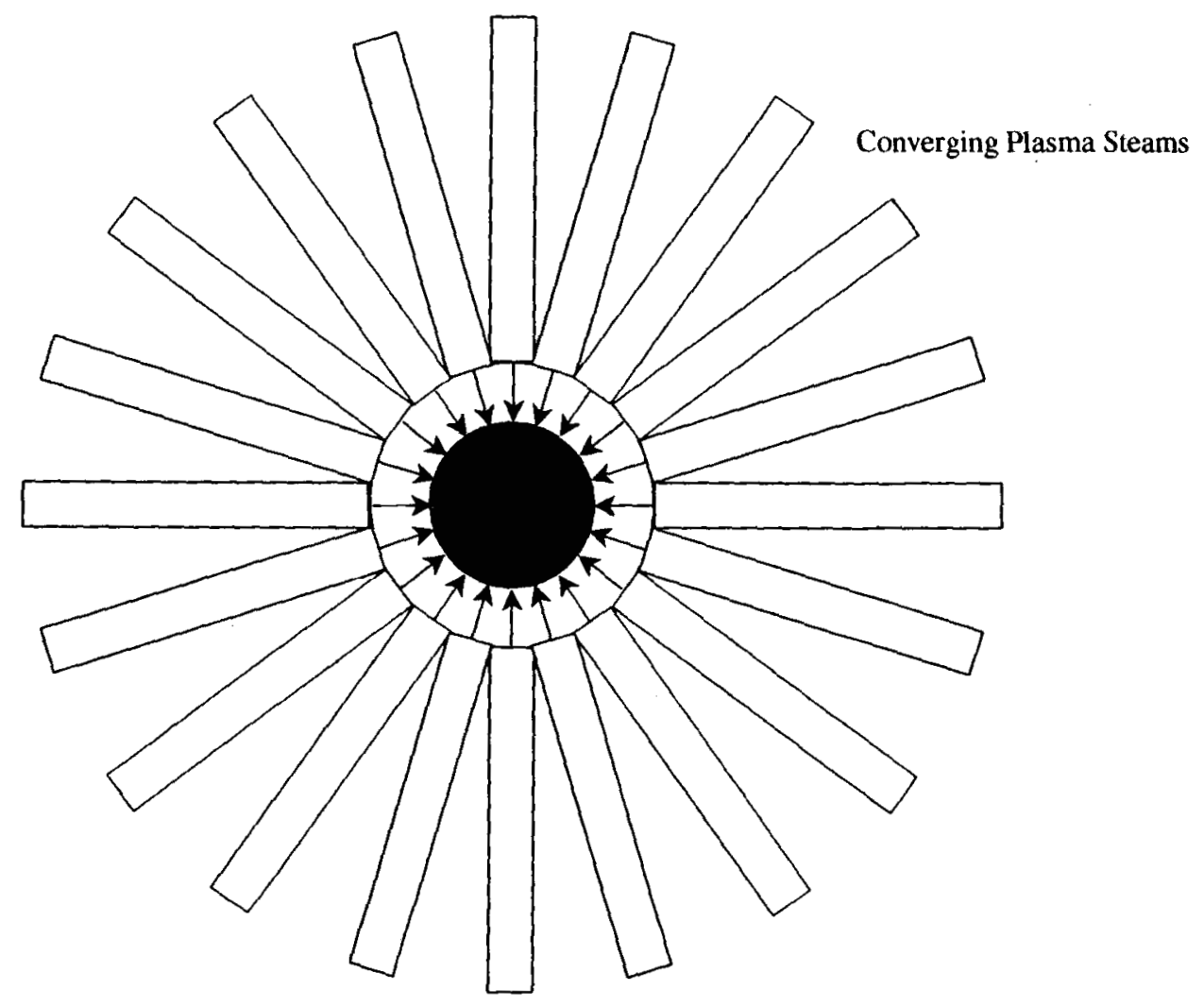

FIGURE 1. Plasma Streams Converging on Target Plasma 
Figure 2 shows a single plasma stream in greater detail. The leading portion, proportionately exaggerated in the diagram for clarity, consists of deuterium plasma; the remainder of the stream is comprised of hydrogen plasma.

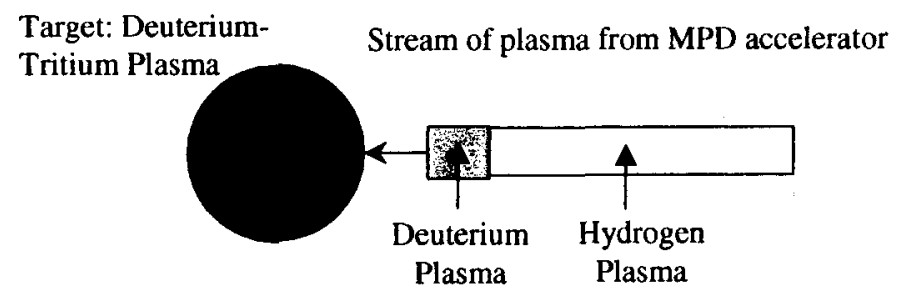

FIGURE 2. Detail of an Individual Plasma Stream

Figure 3a shows the target and surrounding liner, formed when the individual plasma streams coalesce into a shell. The inner and outer liners, composed respectively of deuterium and hydrogen plasma, are shown (again the deuterium region is exaggerated in size). Figure $3 \mathrm{~b}$ shows the point at which conditions in the target initiate deuterium-tritium fusion. Figure $3 \mathrm{c}$ shows the point at which energy release from deuterium-tritium fusion in the target initiates deuterium-deuterium fusion in the inner portion of the liner.

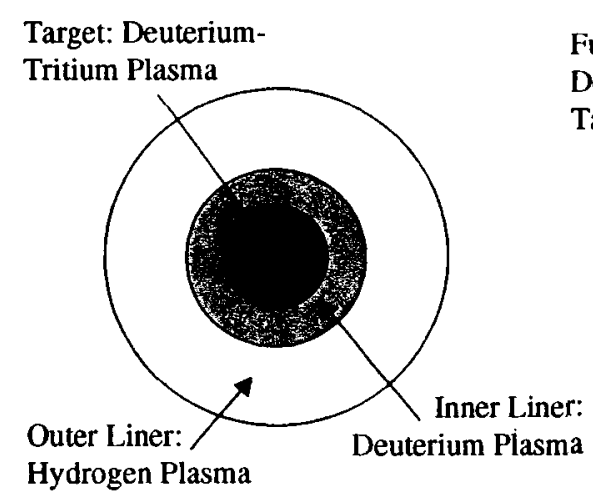

(a) Plasma Compression

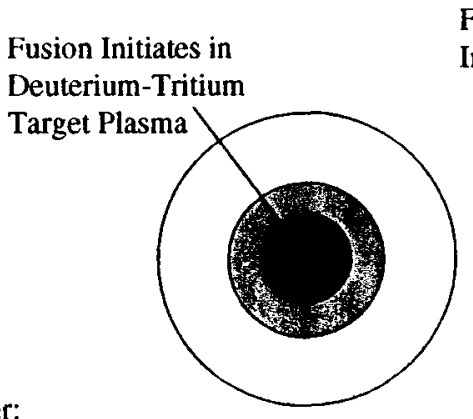

(b) Fusion in Target Region
Fusion Initiates in Deuterium Inner Liner Plasma

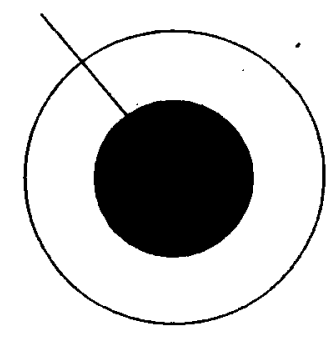

(c) Fusion in Inner Liner

FIGURE 3. Sequence of Events During Compression and Fusion

Finally, Figure 4 shows the hot plasma cloud expanding very rapidly. Fusion reactions in both the central target region and the inner liner have now ceased.

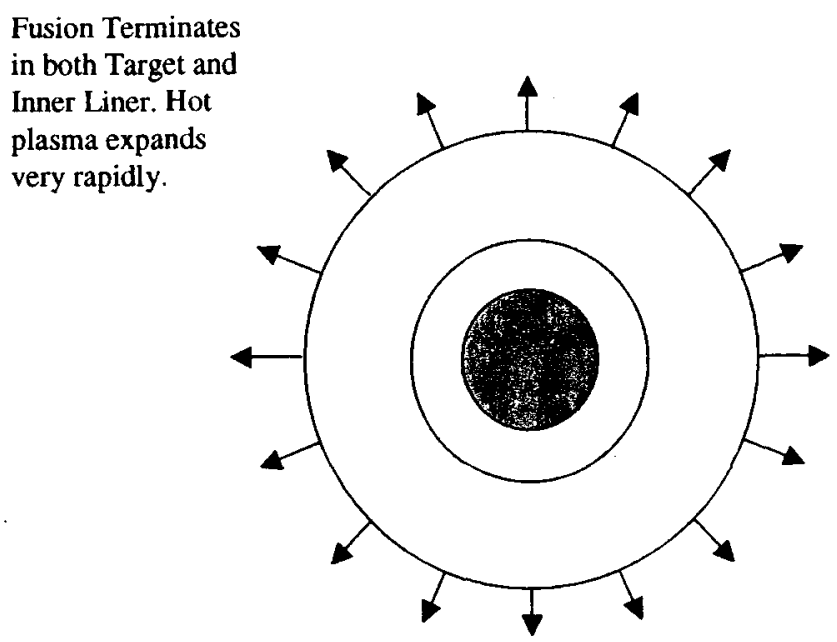

FIGURE 4. Plasma Expansion Phase 
Energy liberated during fusion, both in the target and the inner liner, appears in the form of particle kinetic energy. Charged particle energy is transferred into useful impulse by means of a magnetic field, formed by an array of current carrying coils, which is initially (at the moment of fusion) configured as shown in the left-hand portion of Figure 5. As the plasma expands, the field lines are deformed, as shown in the right-hand portion of Figure 5.
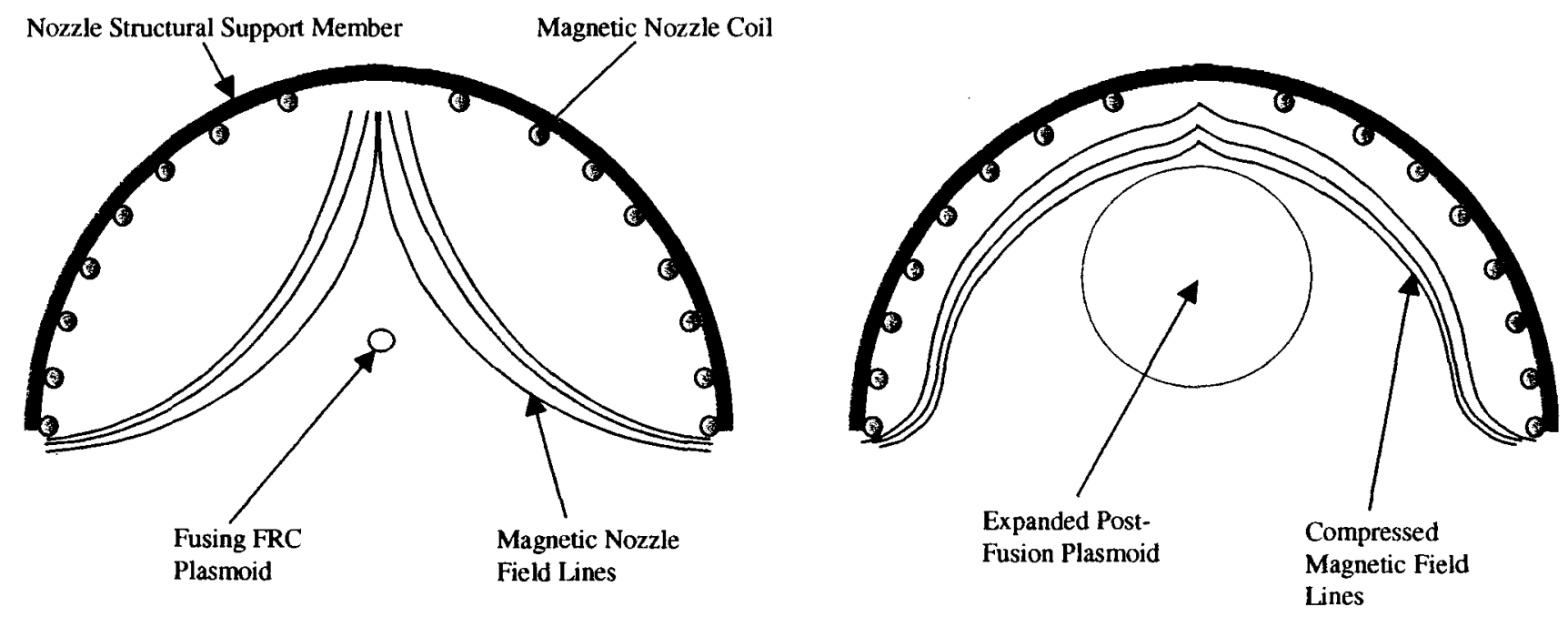

FIGURE 5. Nozzle Magnetic Field Configuration at Fusion and After Plasma Expansion

Distortion and compression of the magnetic field occurs because the plasma, being highly conductive, tends to strongly resist the incursion of any new magnetic flux. Currents are induced within the plasma cloud, which serve to generate a magnetic field which, within the plasma, cancels that due to the external coils. Outside the plasma, the field produced by these induced currents reinforces that due to the coils. Hence, as it expands, the plasma cloud appears to sweep the magnetic field ahead of itself, compressing it and forcing it back towards the nozzle coils.

The expanding plasma cloud's initial kinetic energy is transferred into potential energy in the compressed and deformed magnetic field. Note that the plasma expands freely through the (lower) open portion of the nozzle; no useful impulse is obtained from this part of the expansion.

When the plasma expansion ceases, the deformed and compressed magnetic field springs back towards its initial configuration, in the process expelling the remains of the cloud. During both the compression and expansion phases of magnetic field distortion, a very significant force is transferred to the vehicle, via the nozzle coils.

Electrical energy required to power the next pulse (i.e. to power the plasma guns and to create the initial magnetic field) is extracted by tapping off some of the current induced in the coils during the plasma cloud expansion. The energy required for each plasma gun is stored in a capacitor, attached to the gun itself. The energy required to reestablish the magnetic field, prior to the next fusion pulse, is stored in a Superconducting Magnetic Energy Storage (SMES).

\section{TARGET GENERATION}

In order to achieve a successful fusion burn, the deuterium-tritium target plasma must be located at the precise focus of the MTF nozzle; this is the point at which the entire array of plasma guns is aimed. To overcome the problems of both creating and accurately positioning the target plasma, the following process is adopted. A pair of conical theta pinches, located in diametrically opposite positions on either side of the focus (see Figure 6), is used to create and launch a pair of spheromak plasmoids towards the focus. The two spheromaks, which are 
identical in all respects, except that their torroidal magnetic fields are oriented in opposite directions, collide and merge at the focus. It should be noted that, because of its minimum energy and self-generating magnetic field properties, the spheromak configuration has a relatively long life and will persist until the merging takes place. After merging is complete, the resulting plasma entity will be a Field Reverse Configuration (FRC) plasmoid.

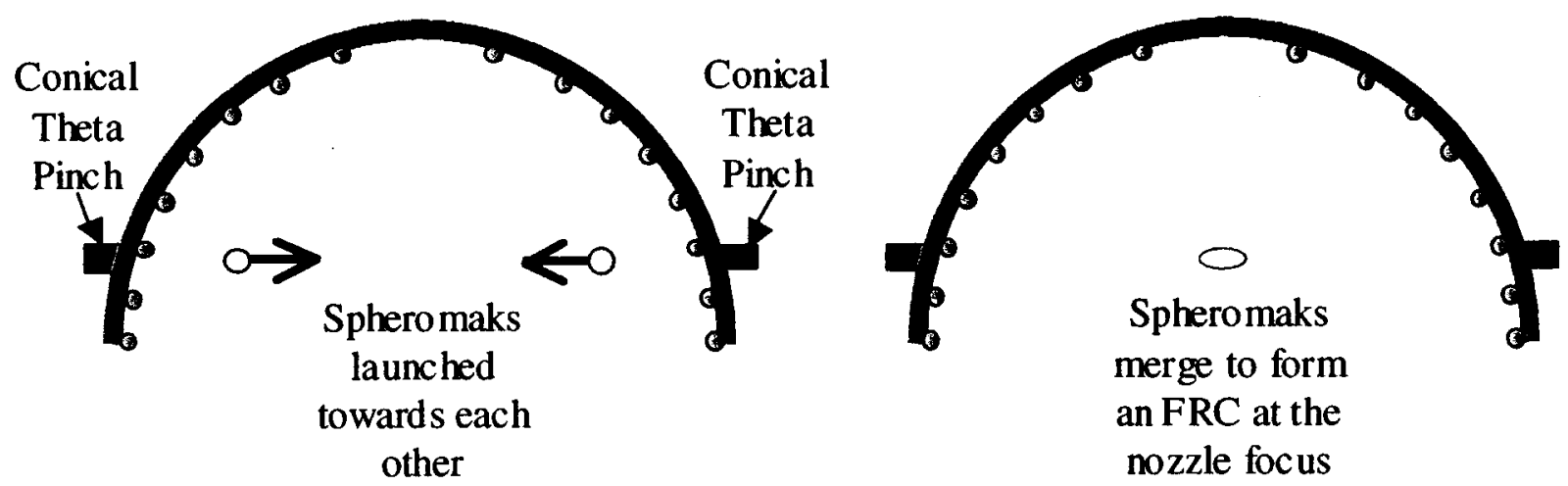

FIGURE 6. Conical Theta Pinches, Spheromak Creation and Merging to form an FRC Plasmoid

Figure 7 shows a cross section through an individual spheromak plasmoid. Although there are both torroidal and poloidal magnetic fields, only the latter extend to the plasmoid surface.

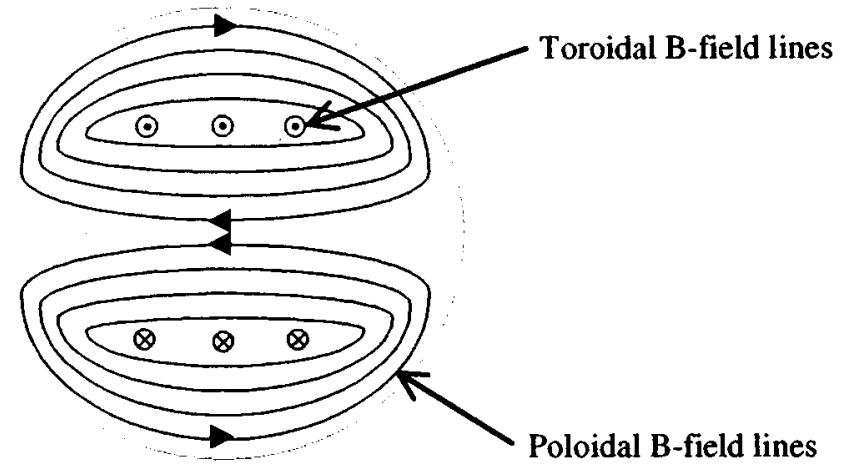

FIGURE 7. A Spheromak Plasmoid

Figure 8 shows the two spheromaks approaching each other. They are identical in every way except that their torroidal magnetic fields are oriented opposite to each other.

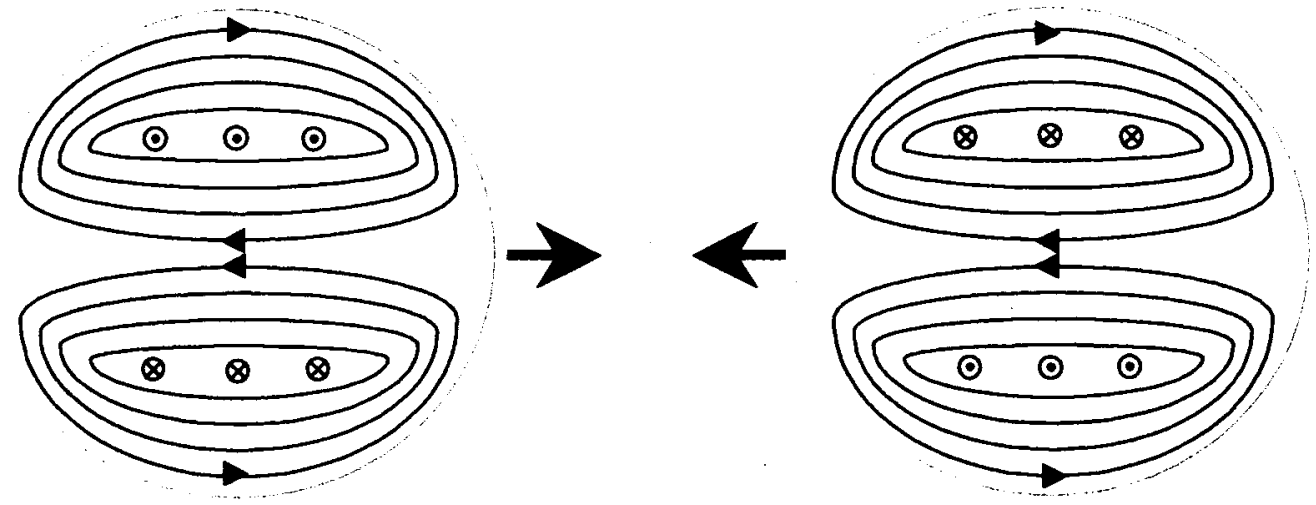

FIGURE 8. Two Spheromaks About to Merge - Note Opposing Torroidal Magnetic Field Directions 
Finally, Figure 9 shows the single FRC plasmoid formed as a result of the two spheromaks having merged. Note that the torroidal magnetic fields of the parent spheromaks exactly cancel out, leaving a single plasmoid with solely poloidal field lines - an FRC.

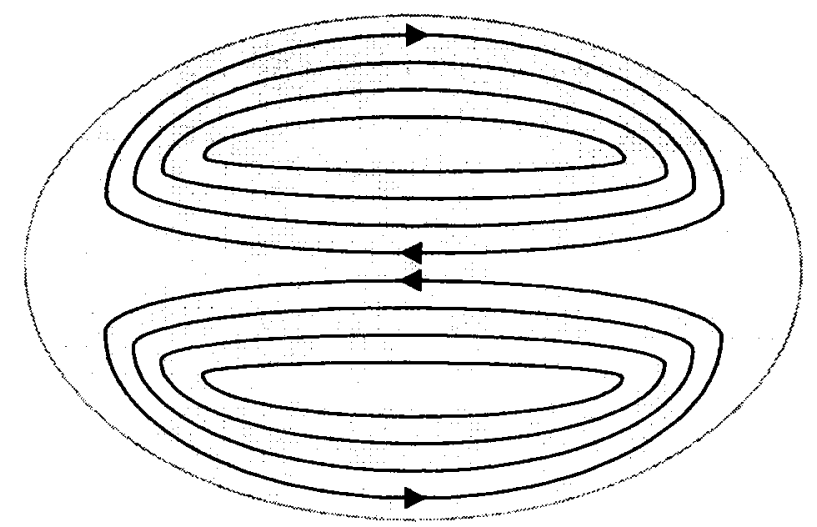

FIGURE 9. Field Reverse Configuration Plasmoid Formed From Two Merged Spheromaks

\section{PLASMA GUN SYSTEM}

Shortly after FRC creation, the plasma streams arrive from the plasma guns and compression commences. It is clearly not possible to compress the FRC isotropically. The obvious requirement for a nozzle exit, to allow the fusion products to exit the MTF device, precludes mounting plasma guns in this region. With no plasma streams incident from below, any incident from above would have the effect of prematurely pushing the FRC out through the nozzle exit. As this is undesirable, there are no plasma guns located around the top of the nozzle. This leaves a band available, centered around an imaginary "equatorial" region of the nozzle, as illustrated in Figure 10.

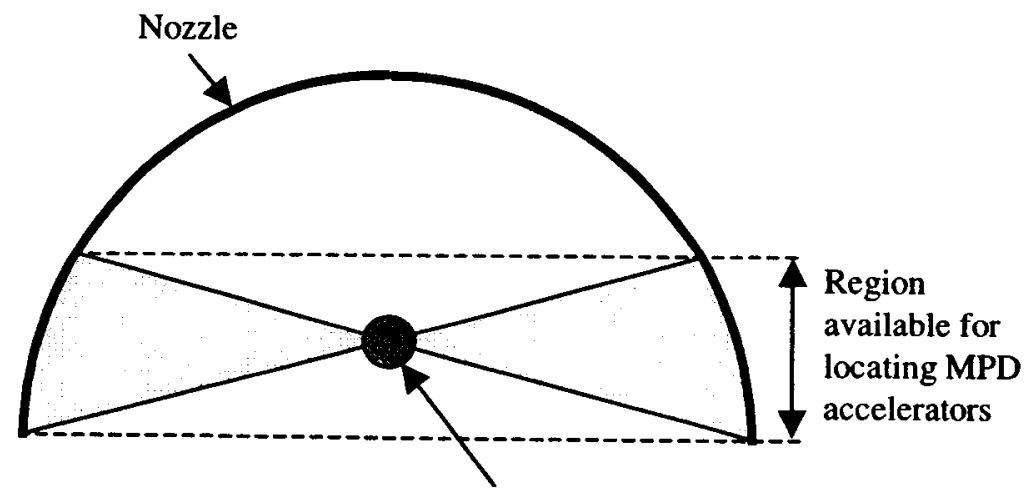

FRC

FIGURE 10. Region of MTF Nozzle Available for Plasma Gun Location

By locating the plasma guns within this "band" and ensuring that they are distributed symmetrically about the nozzle focus - which is the location of the target plasmoid at compression - one can ensure that there is no net momentum imparted to the FRC. 
By restricting plasma gun placement to within the allowable region, it is clear that the plasmoid will not be uniformly compressed over its entire surface by the converging plasma streams. Fortunately this does not impede the compression dynamics. The FRC has the useful property that, when its periphery is compressed in one region, the resulting effect tends to shrink the magnetic field globally. As the magnetic field is "frozen" into the highly conductive plasma, this means that the entire FRC is compressed. By arranging for plasma streams to impact the FRC in the region shown in Figure 10, one can ensure that the entire FRC is compressed.

The plasma gun is illustrated conceptually in Figure 11. The basic device consists of two concentric electrodes, which are connected to a high-energy capacitor. Gas is introduced into the annular gap between the electrodes and the switch is closed. This allows the capacitor to establish a high potential difference between the inner and outer electrode. The potential gradient between the electrodes causes breakdown and ionization of the gas, which completes the circuit and allows the capacitor to discharge.

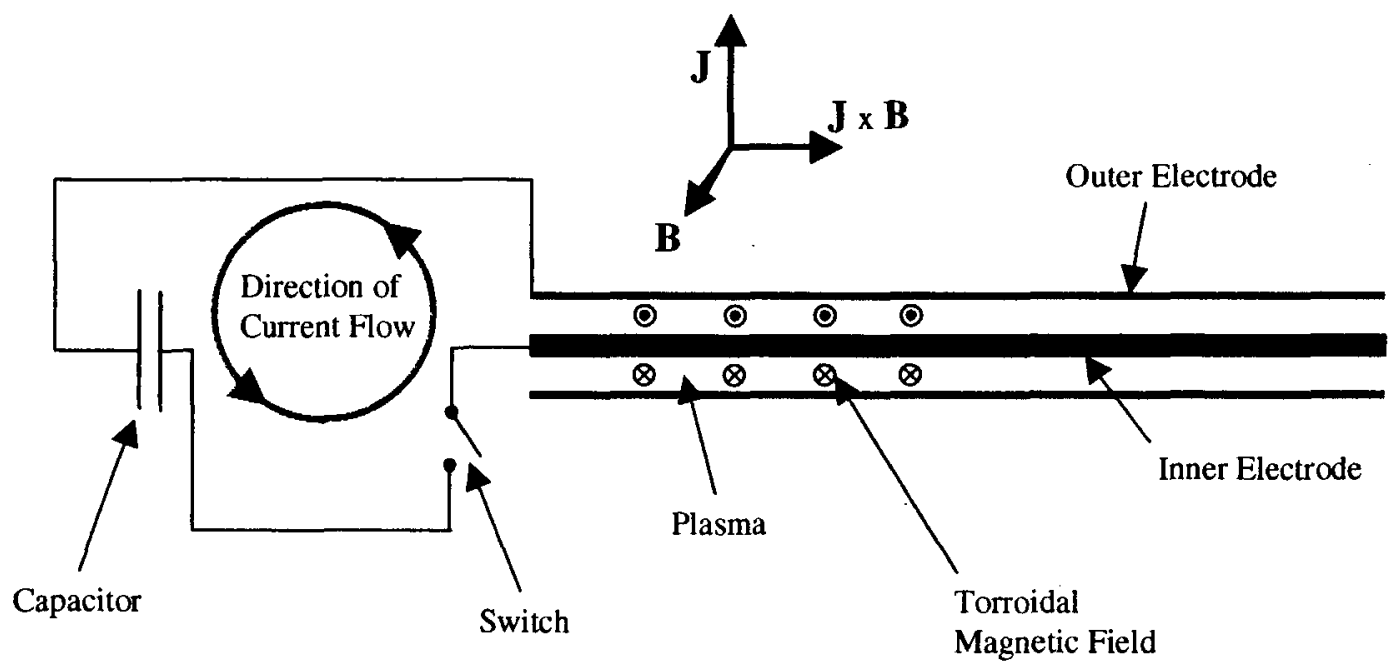

FIGURE 11. Plasma Gun Schematic Design

The direction of current flow through the completed circuit gives rise to a self-generated magnetic field as illustrated in Figure 11. The field lines are oriented torroidally around the inner electrode. This self-generated field is everywhere perpendicular to the direction of current flow, which is, in this example, shown passing radially outwards from the inner to the outer electrode. This gives rise to a $\mathbf{J} \times \mathbf{B}$ force on the plasma (where $\mathbf{J}$ and $\mathbf{B}$ denote current density and magnetic field strength respectively), as shown in Figure 11, which forces it out of the device at very high speed. Note that, as the primary element of the plasma current is due to electron motion, it is the electrons, which first experience the major part of the electromagnetic acceleration force. However, the very strong electrostatic forces, which are generated within the plasma as soon as any charge separation begins to occur, ensure that the ions are accelerated along with the electrons. The fact that it is a quasi-neutral plasma that is accelerated out of the device is significant in that it reduces concerns over the effect of space-charge limitations on the subsequent behavior of the plasma stream.

The actual design of the plasma gun is rendered slightly more complex by the need to focus the plasma stream and counteract its natural tendency to diverge due to gas pressure. The solution to this problem is illustrated in Figure 12, which shows the accelerator portion of the device in greater detail and with greater realism than Figure 11. Each plasma gun is approximately one meter in length without the attached capacitor $(1.4 \mathrm{~m}$ with the capacitor) and has a maximum barrel diameter of 0.25 meters. The barrel and capacitor are fitted together using a scyllac fitting to reduce impedance mismatch. 


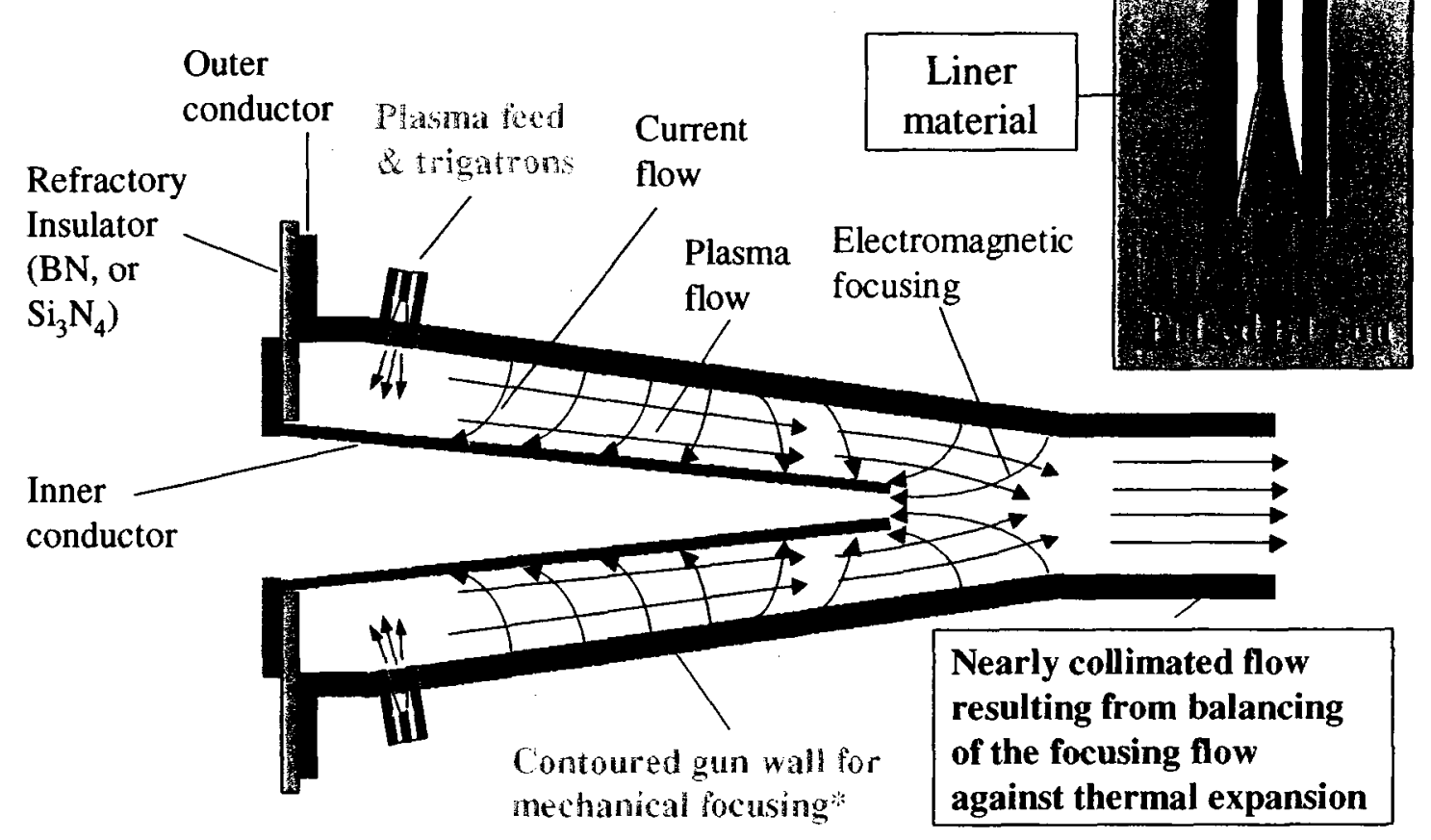

FIGURE 12. Plasma Gun Barrel Design

The converging two-barrel arrangement, with each barrel contoured internally, achieves the necessary focusing effect. Barrel shape must be such that the combination of electromagnetic and thermal expansion forces equal each other. This results in a nearly collimated high-speed gas flow.

The MTF plasma gun system consists of a total of forty-eight MPD accelerators, all positioned around the magnetic nozzle and directed towards its focus, as shown in Figure 13. The forty-eight plasma guns are divided into three banks. The upper bank, with twelve guns, is located above the nozzle focus. The middle bank, with twenty-four guns is level with the focus. The lowest bank, with twelve guns, is located below the focus.

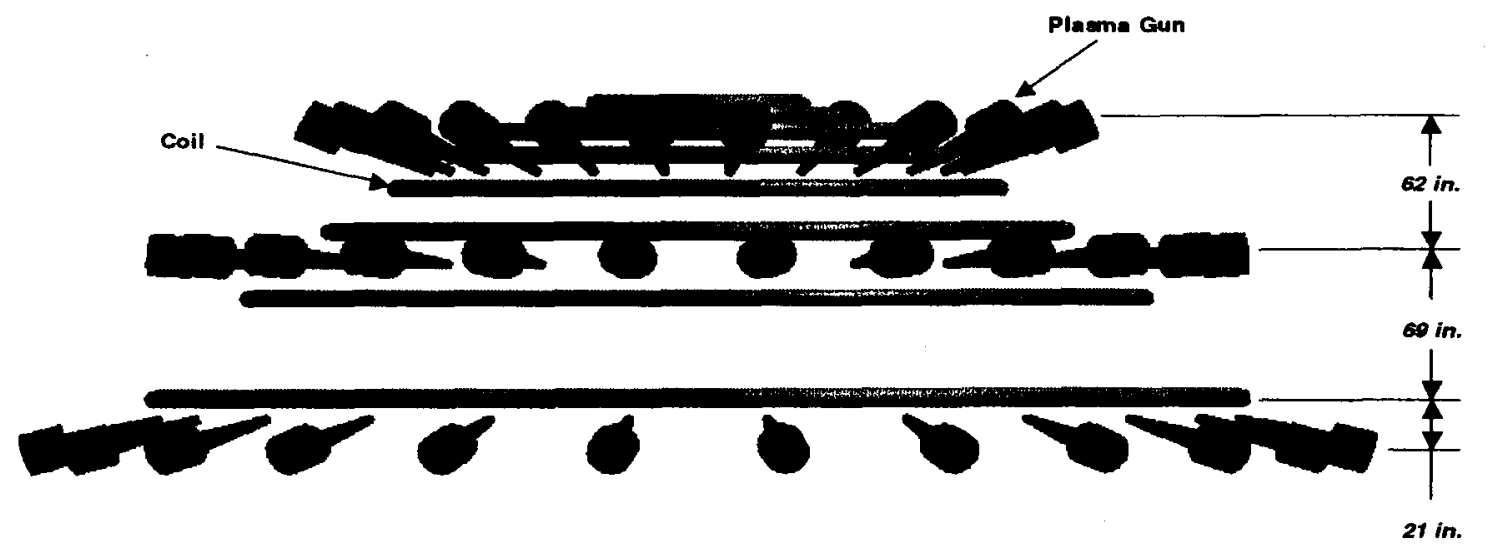

FIGURE 13. Location and Orientation of Plasma Guns Relative to the Coils 
Each plasma gun has its own individual capacitor to provide electrical power during discharge. Each gun delivers a collimated plasma beam at a velocity of $125 \mathrm{~km} / \mathrm{s}$. As explained earlier, the small leading portion of each beam consists of deuterium plasma; the remainder consists of hydrogen plasma.

Note that when the plasma guns are discharged to produce the converging plasma streams aimed at the target, the nozzle magnetic field is not present. Only after the plasma streams are near to the target is the nozzle field initiated. This is to prevent the magnetic field from interfering with the plasma stream trajectories.

\section{MAGNETIC FIELD COILS}

The nozzle magnetic field performs two essential functions. First it acts as a shock absorber, taking the momentum of the expanding plasma cloud and transmitting it to the vehicle as a thrust force. Second, by slowing the expanding plasma cloud and then redirecting it back out of the nozzle, the magnetic field protects the structure and physical components of the nozzle from the extremely high temperatures and from direct damage due to charged particle impacts. The current carrying coils, responsible for producing the magnetic field, are the primary nozzle components.

Eight single turn coils are placed in a parabolic arrangement around the nozzle (see Figure 13). This configuration produces parabolic field lines whose focus coincides with the location of the fusion event. In order to close off the vertex of the magnetic field parabola, and to prevent plasma from passing up along the axis, a single reversed conical theta pinch is installed at the vertex of the nozzle. Each of the eight coils, and the reversed conical theta pinch, is actually composed of two separate coil assemblies: an inner seed field coil and an outer thrust coil.

An applied current in the seed coils, immediately prior to the fusion event, produces the initial "seed" magnetic field. As the plasma cloud expands, post-fusion, it deflects and compresses the seed field - the highly conducting plasma closely approximates the frozen field-line model and thus resists incursion of the field. Plasma cloud expansion also induces very large currents in the outer thrust coils (also referred to as the main coils). These currents interact with the magnetic field, which strengthens as it is compressed by the expanding plasma cloud, to produce a very large $\mathbf{J} \times \mathbf{B}$ force on the main coils.

\section{Seed Coils}

Each seed field coil consists of a super conducting $\mathrm{YBa}_{2} \mathrm{Cu}_{3} \mathrm{O}_{7}$ single turn loop that is energized by the SMES via a coaxially arranged super conducting power bus (of which further details are given later). The seed field coil is cooled with cryogenic liquid nitrogen, which flows through the conductor cable mesh. Super conducting coils are used to minimize the power loss due to ohmic heating. Once the seed field coils are energized, they produce the initial field within the nozzle.

\section{Main Coils}

Each main or thrust coil is laid coaxially over its associated seed field coil and, unlike the seed field coil, makes a continuous single turn loop. Because the main coil operates at high temperature, it is comprised of a thin layer of Titanium Diboride and Molybdenum $\left(\mathrm{TiB}_{2} / \mathrm{Mo}\right)$ metal matrix composite. This material has a conductivity comparable with that of copper, and a melting temperature in excess of $3000 \mathrm{~K}$. The thickness of the conductor is set in order that the relatively slowly changing magnetic flux of the seed field coil can pass through it, but the more rapidly changing compressed flux is completely absorbed. The main coil is responsible for transforming the compressed magnetic flux inside the nozzle into a thrust force. This is accomplished by the Lorentz forces generated from the large magnetic field inside the nozzle and the very large (Mega-Amp level) currents induced in the main coils by the compressed magnetic field. 


\section{Coil Structural Design}

Between the large oscillatory impulses of the fusion events and the magnetic hoop stresses in the coils, the nozzle must withstand high dynamic loading conditions. Most of these forces are coupled to the structure through the coils which, as a result, must be structurally robust. This requirement along with the dielectric and thermal requirements of the coils makes material selection difficult. To solve these issues, advanced composites and ceramics must be used; their superior strength, dielectric properties, and high melting temperatures allow for strong lightweight components. Also, because they can be laid up together, their use should reduce the need for complex machining.

Ceramics are, however, extremely brittle and cannot withstand significant dynamic loading. Although they show exceptional strength against compressive loads, they have very low tensile strength. For this reason, it is important to lay up these materials in such a manner that the loads can be transferred to an outer carbon-carbon composite that does have excellent tensile strength. A typical coil cross-section is shown in Figure 14 and its corresponding dimensions given in Table 1. 


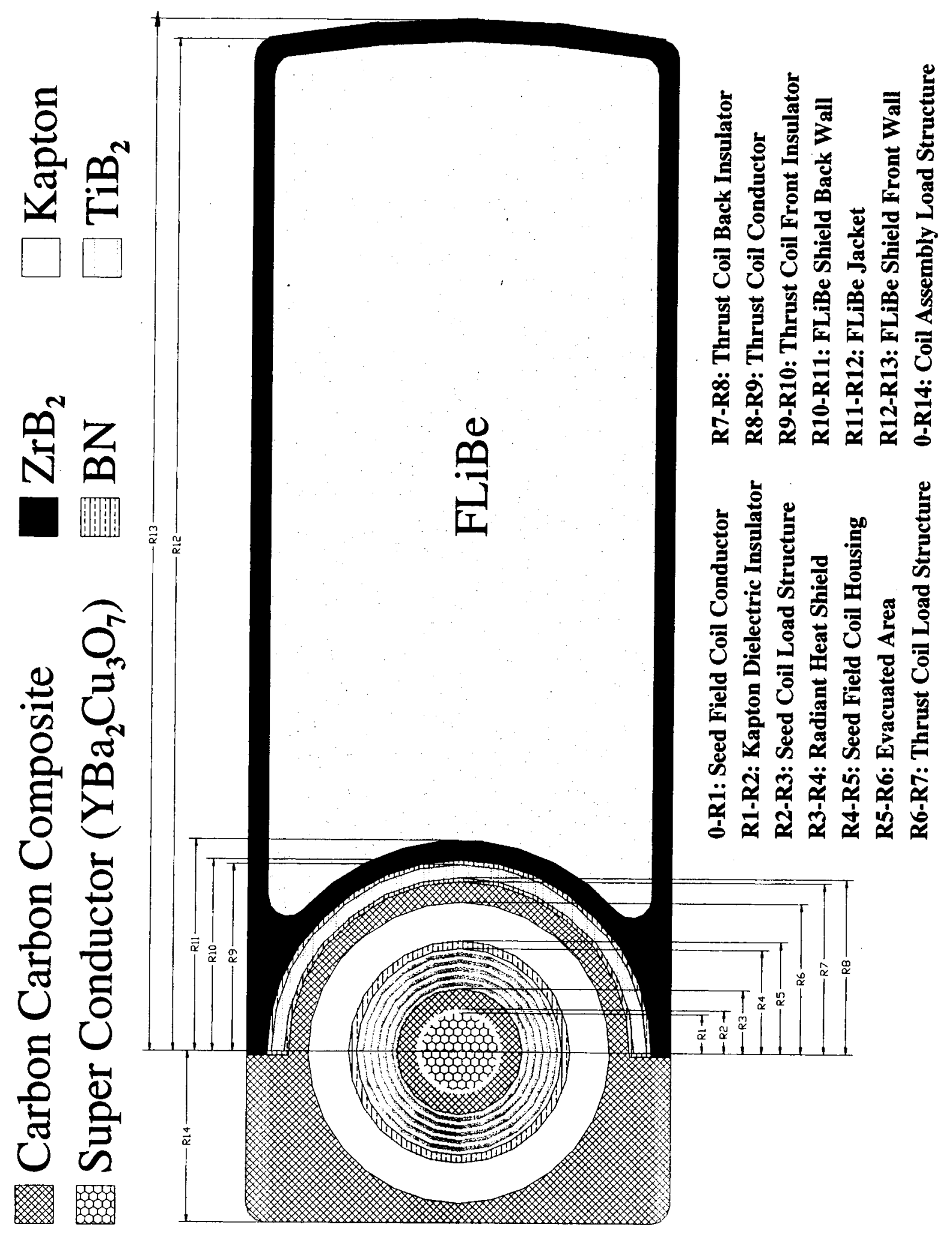

Figure 14. MTF Nozzle Coil Assembly Cross Section 
Table 1. MTF Nozzle Coil Assembly Dimensions

\begin{tabular}{|c|c|c|c|c|c|c|c|c|c|c|c|c|c|c|}
\hline & & & & & & & & & & & & & & \\
\hline 1 & 0.33 & 0.43 & 0.93 & 1.93 & 2.13 & 3.13 & 3.63 & 3.73 & 3.77 & 3.87 & 4.37 & 24.37 & 4.87 & 3.63 \\
\hline 2 & 0.37 & 0.47 & 0.97 & 1.97 & 2.17 & 3.17 & 3.67 & 3.77 & 3.83 & 3.93 & 4.43 & 24.43 & 24.93 & 3.67 \\
\hline 3 & 0.42 & 0.52 & 1.02 & 2.02 & 2.22 & 3.22 & 3.72 & 3.82 & 3.89 & 3.99 & 4.49 & 24.49 & 24.99 & 3.72 \\
\hline 4 & 0.47 & 0.57 & 1.07 & 2.07 & 2.27 & 3.27 & 3.77 & 3.87 & 3.97 & 4.07 & 4.57 & 24.57 & 25.07 & 3.77 \\
\hline 5 & 0.54 & 0.64 & 1.14 & 2.14 & 2.34 & 3.34 & 3.84 & 3.94 & 4.06 & 4.16 & 4.66 & 24.66 & 25.16 & 3.84 \\
\hline$\overline{6}$ & 0.63 & 0.73 & 1.23 & 2.23 & 2.43 & 3.43 & 3.93 & 4.03 & 4.19 & 4.29 & 4.79 & 24.79 & 25.29 & 3.93 \\
\hline 7 & 0.76 & 0.86 & 1.36 & 2.36 & 2.56 & 3.56 & 4.06 & 4.16 & 4.39 & 4.49 & 4.99 & 24.99 & 25.49 & 4.06 \\
\hline 8 & 0.97 & 1.07 & 1.57 & 2.57 & 2.77 & 3.77 & 4.27 & 4.37 & 4.72 & 4.82 & 5.32 & 25.32 & 25.82 & 4.27 \\
\hline
\end{tabular}

A typical coil cross-section is described as follows - refer to Figure 14. At the center of the coil assembly is the seed field coil conductor. This consists of a mesh of super conducting material, using a 3:1 conductor to coolant gap ratio. This allows adequate cryogenic coolant to flow along the conductor and still remain below its critical temperature. A layer of Kapton dielectric material surrounds the conducting mesh. This material serves as an electrical insulator and prevents arcing within the coil. The current through the seed field coil will cause it to undergo large hoop stresses due to the magnetic pressure within the loop. Because the current is pulsed, the seed coil will undergo a large number of stress cycles during operation. A layer of carbon-carbon composite is wrapped around the thin layer of Kapton to prevent strain on the seed field coil. Outside the carbon composite are five layers of low emissivity polished aluminum, each $1 \mathrm{~mm}$ in thickness and separated by $0.8 \mathrm{~mm}$ thick evacuated regions. These alternating layers serve as a radiant energy heat shield and prevent heat from the thrust coil from penetrating into the seed field coil. The coaxially layered aluminum layers are electrically continuous so that no net current can be induced in them by the varying seed field flux. This assembly is covered with a thin layer of boron nitride to serve as a lightweight high temperature casing.

The thrust coil assembly surrounds the seed field assembly, the two being separated by an evacuated section 0.5 $\mathrm{cm}$ in thickness. This further reduces heat conduction from the high temperature thrust coil into the cryogenictemperature seed field coil. Beyond this evacuated region is the structural carbon-carbon composite support for the thrust coil assembly. Loads from the thrust coil conductor are transferred into this support and thus into the nozzle as thrust. Both the thrust and seed field coils are structurally tied to the main support splines of the magnetic nozzle. The thrust coil conductor forms a "C" shape when viewed in cross section; the convex part of the " $C$ " pointing towards the central axis of the nozzle. Since there is no magnetic flux compression outside the MTF nozzle, there is no need for a conductor on the outer side (i.e. furthest from the nozzle central axis) of the coil assembly. The high temperature $\mathrm{TiB}_{2} / \mathrm{Mo}$ thrust coil conductor is completely surrounded by a layer of boron nitride. In addition to being a high temperature ceramic, boron nitride has excellent dielectric properties and can prevent arcing from within the coil.

To complete the thrust coil assembly, a molten salt coolant jacket is placed between the thrust coil conductor and the focus of the nozzle. This coolant jacket is made from zirconium diboride $\left(\mathrm{ZrB} \mathrm{B}_{2}\right)$, a high temperature ceramic that is not prone to reaction with the molten salt coolant.

\section{MAGNETIC NOZZLE STRUCTURE}

As shown in Figure 15, the coil assemblies, target generators and plasma guns are all mounted on a structural framework, which serves to hold the various components in their correct location and also transmits the thrust force to the remainder of the vehicle. The structure consists of twelve equally spaced, tapered splines, each of which extends from very near the apex of the device down to some distance below the focus. In keeping with the nozzle profile, each spline has a near-parabolic profile. The structure is completed by four rings, as shown in Figure 15. Both the splines and the rings are constructed of a carbon-carbon composite. 


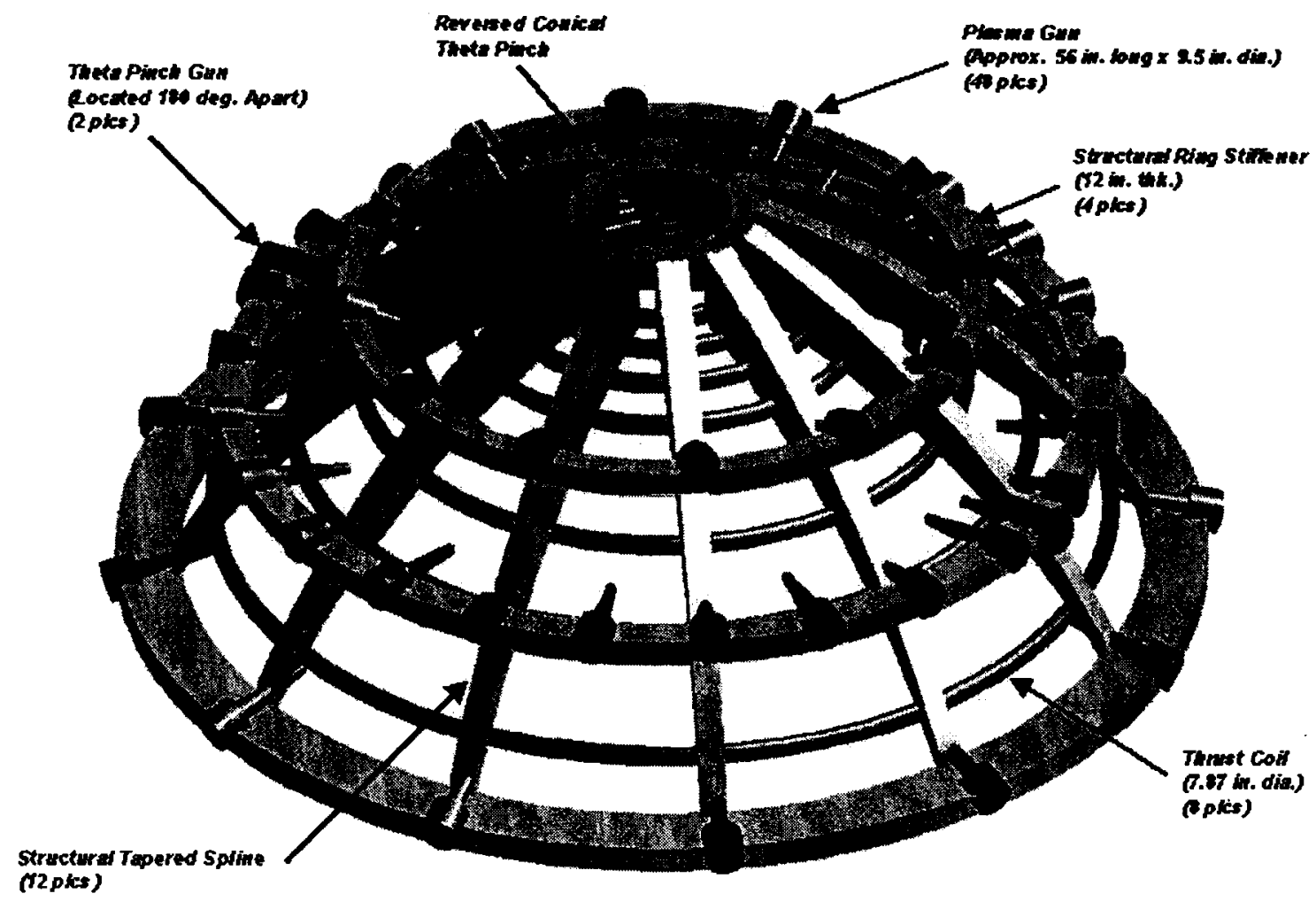

FIGURE 15. Location of Major Components

\section{NEUTRON PROTECTION}

The deuterium-tritium fusion reaction, which takes place in the target plasmoid, can be written as follows (Bond, 1978)

$$
{ }^{2} \mathrm{D}+{ }^{3} \mathrm{~T} \rightarrow{ }^{4} \mathrm{He}(3.5 \mathrm{MeV})+{ }^{1} \mathrm{n}(14.1 \mathrm{MeV})
$$

where ${ }^{2} \mathrm{D},{ }^{3} \mathrm{~T}$ and ${ }^{4} \mathrm{He}$ denote deuterium, tritium and helium-4 nuclei respectively, and ${ }^{1} \mathrm{n}$ denotes a neutron. The reaction product energies are indicated in parenthesis.

The deuterium-deuterium fusion, which takes place in the inner portion of the plasma liner, can proceed according to two different reactions, which can be written as follows (Bond 1978)

$$
\begin{aligned}
& { }^{2} \mathrm{D}+{ }^{2} \mathrm{D} \rightarrow{ }^{3} \mathrm{~T}(1.01 \mathrm{MeV})+{ }^{1} \mathrm{p}(3.02 \mathrm{MeV}) \\
& { }^{2} \mathrm{D}+{ }^{2} \mathrm{D} \rightarrow{ }^{3} \mathrm{He}(0.82 \mathrm{MeV})+{ }^{1} \mathrm{n}(2.45 \mathrm{MeV})
\end{aligned}
$$

where ${ }^{1} \mathrm{p}$ and ${ }^{3} \mathrm{He}$ denote a proton and a helium-3 nucleus respectively.

The charged particle products of both the central and liner reactions can be deflected by the nozzle magnetic field and thus impart useful impulse to the vehicle without physical contact. However the neutrons, from both the core deuterium-tritium and the liner deuterium-deuterium reactions, being electrically neutral, cannot be deflected. Despite the relatively open design of the nozzle lattice, which permits a significant proportion of the 
neutrons to escape, those which do still impact present one of the principal design challenges for an MTF propulsion system.

The challenge manifests itself in several ways. First it is necessary to protect the nozzle (including the structure, coils, plasma guns and other components) from the physical damage which would otherwise result from highenergy neutron bombardment. Secondly some radioactivity is induced in the MTF structure. This can arise because slow neutrons, upon striking the nozzle, may be absorbed by the nuclei of those atoms which compose the structure, thus rendering them radioactive. Thirdly, as the neutrons, particularly those originating from the central deuterium-tritium reactions, have very high energies, they deposit a significant amount of thermal energy into the nozzle when they impact. Removing this very large heat flux, and limiting the nozzle material temperatures in order to maintain mechanical properties, are major challenges.

In mitigation, we note that a significant degree of neutron moderation is likely to take place in the outer regions of the plasmoid. Immediately following fusion, when the thermonuclear neutrons are traveling outwards from the central regions, the density of the surrounding layers of compressed hydrogen will be very great. It is likely that a significant amount of neutron moderation will take place within this region due in part to the high density and in part to the excellent moderating capabilities of hydrogen, the dominant nucleus present. A full analysis of this process has yet to be conducted. Accordingly a somewhat pessimistic assumption of only $20 \%$ moderation has been made for the purposes of the HOPE design study. At some later stage, when the necessary moderation analysis has been conducted, it should be possible to favorably revise the MTF design. Both neutron shielding and thermal control system masses should decrease.

Neutrons can cause damage to materials in a variety of ways. Their total integrated flux, defined as the fluence, must be limited to prevent damage. High-energy neutrons normally pass through most materials without any atomic collisions simply because of the relatively small size of the nucleus. However, where collisions do occur, they can result in atomic displacements, where the atom is effectively driven out of its location within the crystal matrix. This phenomenon is referred to as neutron embrittlement and can be thought of as a microscopic strain hardening mechanism. If a large enough number of these events occur, as is the case in high radiation environments, the material will loose many of its structural, electrical, and heat conduction properties. Structural elements can fail, heat exchangers can loose performance, and electrical conductors can increase in resistivity. Without appropriate protective steps, all of these reactions would result in a decrease in the performance of the MTF propulsion system.

Low energy neutrons can also produce adverse effects. Neutrons at low energies are more easily absorbed by atomic nuclei, usually resulting in the creation of a radioactive isotope. This too can change material properties and can cause problems with electronic circuitry. Fortunately for most materials there exist a range of energies at which the neutrons are too energetic to be absorbed, but are at low enough energy to pass through without interacting with the nuclei. Within this energy range, the material is effectively transparent to the neutrons.

Designing a neutron radiation shield capable of moderating neutrons with a range of initial energies would be a challenging task. It would probably necessitate the use of a composite shield, consisting of many different layers of materials, with the outer layers slowing down fast neutrons but allowing slower ones to pass to the next layer. Each successive layer would have to repeat this process until all the neutrons were moderated to the appropriate energy. Such a shield would act as a neutron "filter" and would require much less mass than conventional shielding. Unfortunately, the design of such a "tailored" shield was not possible within the scope of the HOPE study. With available techniques, at best, a large population of the high-energy neutrons can be reduced to a level of transparency. The fusion community is currently experimenting with the molten salt FLiBe (a mixture of Lithium Fluoride and Beryllium Fluoride, pronounced "flibe"). FLiBe has a good atomic cross section for slowing neutrons, a high vaporization temperature, and acceptable viscosity.

In addition to its shielding properties, liquid FLiBe can also serve as a primary cooling fluid. This yields a particularly efficient design solution as the material into which the neutron thermal energy is first deposited (the FLiBe) is also the medium which carries it away from the nozzle. The neutron energy must be pumped out 
of the system so that a nozzle operating temperature of no greater than $1,500 \mathrm{~K}$ is maintained. This is in keeping with the maximum operating temperature that the hottest portions of the nozzle can withstand. It also ensures that the FLiBe remains below its vaporization point.

For these reasons, FLiBe was selected as the neutron shield material and active cooling fluid for the MTF nozzle.

\section{MAIN COOLING SYSTEM}

At the focus of the MTF nozzle, the fusion reactions can produce plasma temperatures as high as $\mathbf{3 0 0}$ million Kelvin (the approximate temperature at which deuterium-deuterium fusion occurs). Fortunately cooling, which accompanies the plasma expansion process, and the magnetic fields within the nozzle prevent material at these high temperatures from coming into contact with the structure. These mechanisms, however, do not prevent radiant electromagnetic (Bremsstrahlung) and neutronic radiation from reaching the nozzle structure. If left uncooled, the nozzle would quickly exceed its maximum allowable operating temperature and would melt, primarily on account of the neutron energy; therefore an active cooling system is needed to remove the large (multi-Megawatt) heat input to the structure.

The resulting cooling system employs a complex network of coolant channels, running throughout the MTF nozzle structure and the coils. A variety of high temperature liquid metal and molten salt coolants flow within these channels; these ultimately transfer the heat away from the MTF device and out to a radiator array for (radiative) disposal into space.

As explained earlier, the coolant must serve two purposes. It must carry away heat at a high temperature while remaining a liquid and must also serve as a radiation shield for the nozzle structure. Gamma radiation, when absorbed by a material, simply produces an increase in temperature. Although the heat from the gamma rays must be removed, it causes little damage when compared to that of the neutrons.

FLiBe flows down each of the twelve structural splines (see Figure 15) of the MTF nozzle along cooling channels below the surface facing the nozzle focus; it returns along a manifold located on the outward-facing surface of the spline. FLiBe channels are also placed along the sides of each spline to serve as feed and return manifolds for the cooling channels placed along the structural ring stiffeners and the magnetic coils between each spline. FLiBe flows down the feed manifold on the right side of a spline. This manifold feeds eight coil coolant channels and three ring stiffener coolant channels. All of these feed into the return manifold located on the left side of the adjacent spline; this splits the MTF nozzle into twelve different main circuits. All of these circuits are combined into a common, structurally integrated feed and return manifold found at the interface of the MTF nozzle and vehicle.

High temperature FLiBe leaves the MTF nozzle along the common return manifold and feeds into a heat exchanger which is part of a Brayton power cycle that uses helium as its working fluid. The Brayton system converts some of the heat of the MTF nozzle into mechanical energy, using a gas turbine that drives the power cycle compressor, FLiBe pump, and the cryocooler used with the SMES system. The remaining waste heat is transferred to a radiator via a potassium-sodium working fluid heat exchanger. The cooled FLiBe is sent to the FLiBe pump and returned to the MTF nozzle along the common feed manifold.

The Brayton power cycle heat exchanger also serves an additional function. Since the MTF propulsion system must be shutdown during various phases of the mission (e.g. while at the destination), the heat source that normally keeps the molten FLiBe a liquid will be absent. From an operations perspective, it is undesirable to allow the FLiBe to solidify. The HOPE vehicle utilizes an SP-100 fission reactor to provide non-propulsive power for mission operations. This is a 375-megawatt electrical ( 2 megawatt thermal) output reactor that uses a liquid metal Rankine power cycle. Usually the waste heat from this power system is sent to a separate radiator array. When the MTF propulsion system is inactive, heat from the SP-100 can be sent to the Brayton cycle heat 
exchanger to keep enough heat in the FLiBe so that it will remain a liquid. During these periods, the pump system operates at a lower duty cycle than when the MTF propulsion system is active. The thermal schematics for these systems and for the "hotel" waste heat rejection (i.e. heat rejected from the crew quarters and other elements of the payload) system are shown in Figure 16.
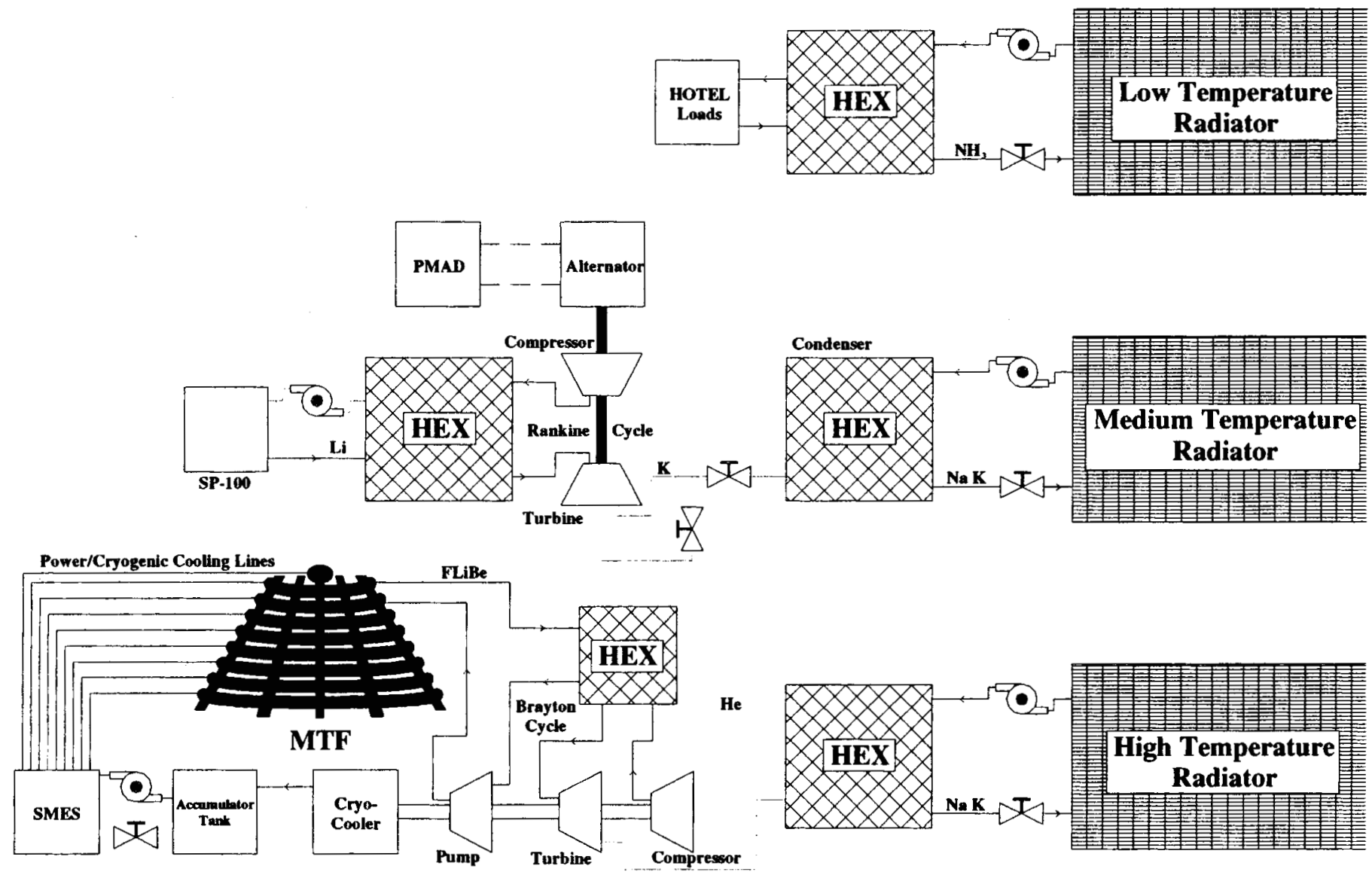

FIGURE 16. MTF Thermal Management Schematic

\section{RECHARGE SYSTEM}

The recharge system is responsible for converting a small portion of the expanding plasma cloud's kinetic energy into usable electric power for the magnetic seed field coils and the plasma guns. The plasma expansion process induces large currents in the thrust coils, some of which can be commutated out of the coils and used for recharging the system. The circuit diagram for this system is shown in Figure 17. 


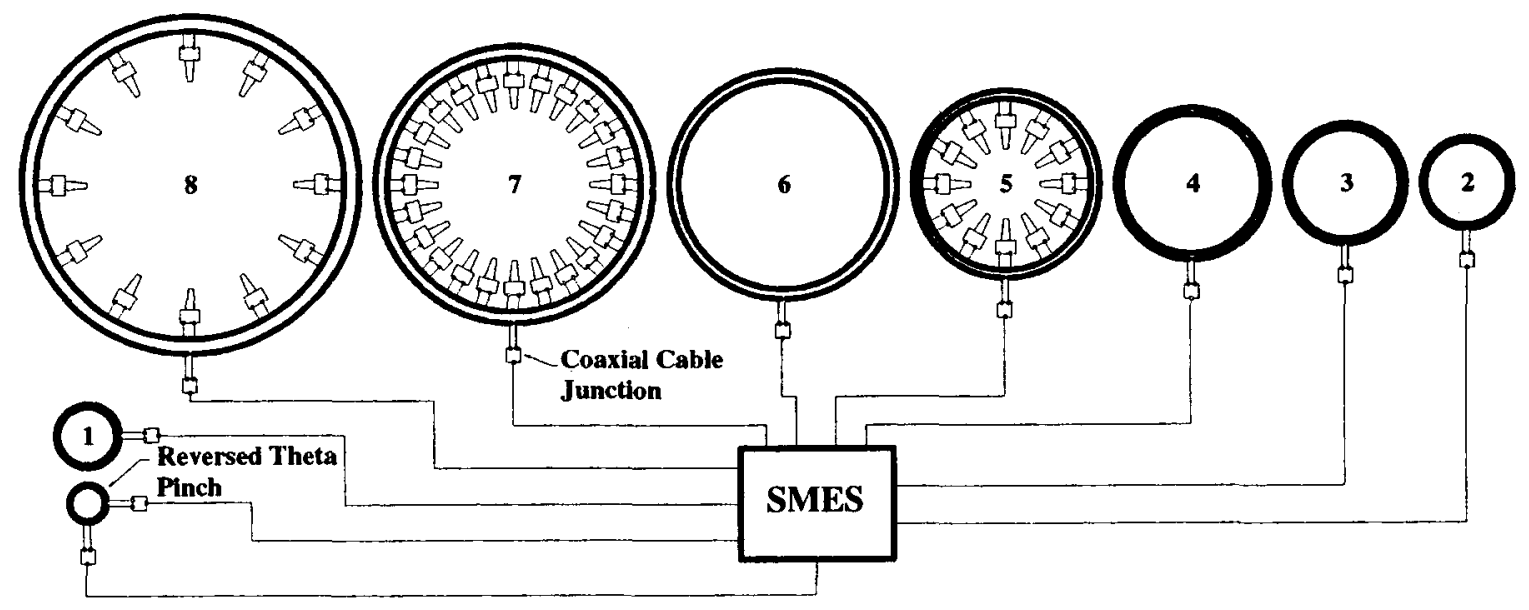

FIGURE 17. Recharge System Schematic

The three plasma gun banks are located along different coils (numbers 8,7 and 5 - where the coils are numbered in order of increasing size). Each individual coil is responsible for charging the bank of plasma guns which it supports. Each plasma gun has its own charging.circuit connected to the thrust coil in such a way that the impedance value of the circuit lines, capacitor, and the section of the thrust coil isolated by the charging circuit terminals are equal. This ensures that only the amount of power needed to charge the capacitor can be tapped off from the thrust coil. The charging circuit requires a high duty switch for isolating the capacitor from the thrust coil. Although a spark gap system can provide the needed performance, life limitations would probably ultimately make an advanced switching system desirable.

As mentioned earlier, the seed field coils draw power from a SMES, which has the capability to accept and discharge large amounts of electrical energy in a very short period of time. The SMES is connected to, and receives power from, the thrust coil conductor of the reversed conical theta pinch, which is located at the vertex of the MTF nozzle. Electrical energy, generated inductively in the thrust coil conductor is transmitted to the SMES. This energy is stored within the SMES in the form of a magnetic field and, at the appropriate time, is transmitted to the seed field coils to produce the initial magnetic field lines inside the MTF nozzle prior to initiation of the next fusion event. This charging circuit is shown in Figure 18. 


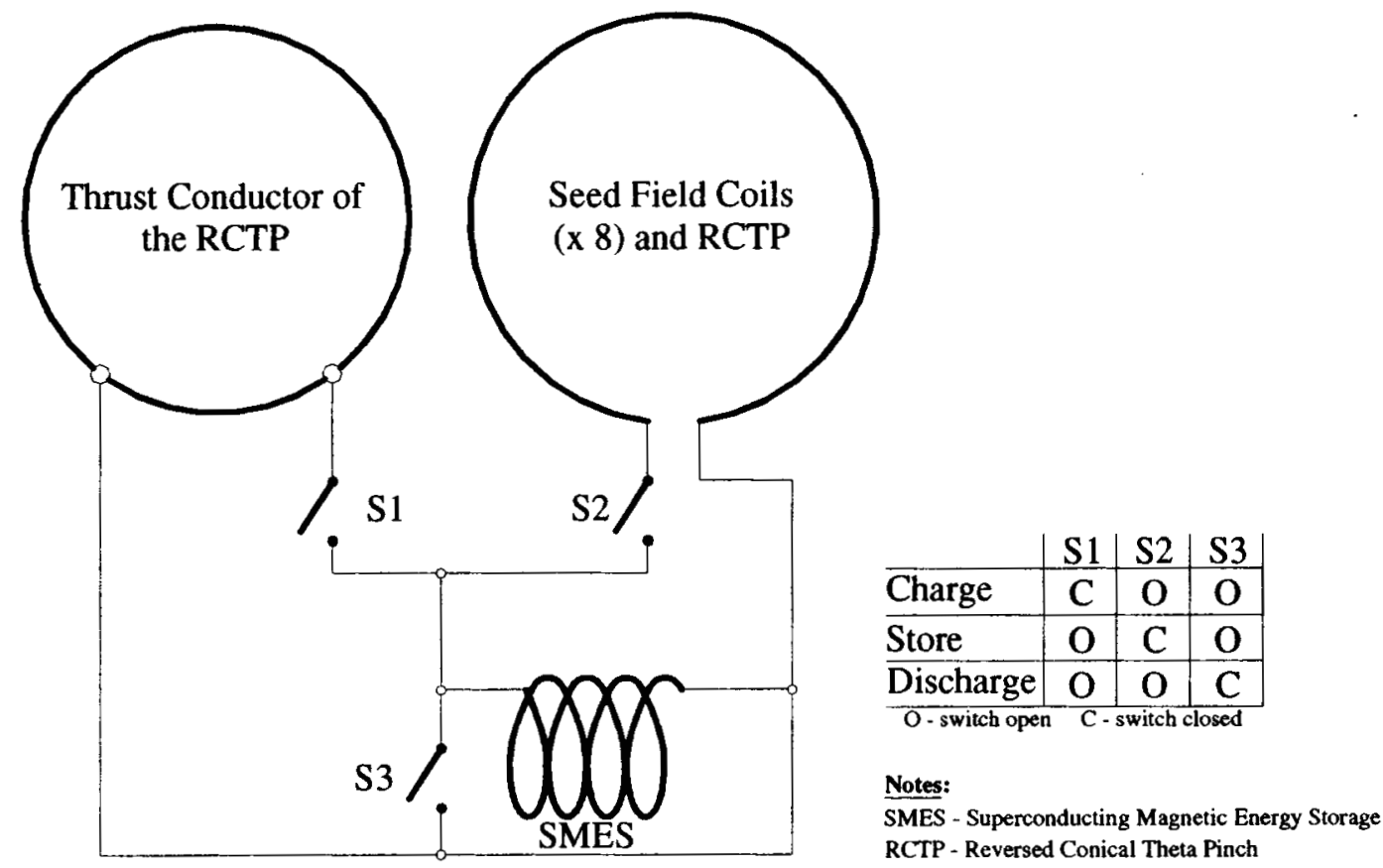

FIGURE 18. SMES Charging Circuit

Power is transmitted to and from the SMES, via super conducting coaxial transmission cables. Since the SMES, transmission lines, and seed field coils are all super conducting, they share a common cryogenic cooling system. Liquid nitrogen is cooled in an accumulator tank by a cryocooler and delivered to the SMES (see also Figure 16), from where it travels down the transmission lines, flows around the seed field coils and finally back up the transmission lines to the SMES and accumulator tank for re-cooling.

Since the transmission lines must both carry and return the cryogen coolant, they require two coolant channels. Because the overall diameter of the transmission lines is not as critical as the coils, a wet mesh arrangement is not used in the interest of minimizing coolant flow frictional losses. This allows one coolant channel for each conductor in the cable. The transmission lines run along the MTF nozzle structure to the different coils in a nonsymmetrical fashion. This could affect the symmetry of the nozzle magnetic field, for which reason coaxially arranged conductors are used to reduce the magnetic field produced along the pulsed transmission lines. A radiant heat shield is also employed on the transmission lines. The resulting transmission line design is shown in Figure 19 with dimensions given in Table 2. 
Kapton Insulator

39 Super Conductor $\left(\mathrm{YBa}_{2} \mathrm{Cu}_{3} \mathrm{O}_{7}\right)$
$\mathrm{LN}_{2}$ Coolant

BN Housing

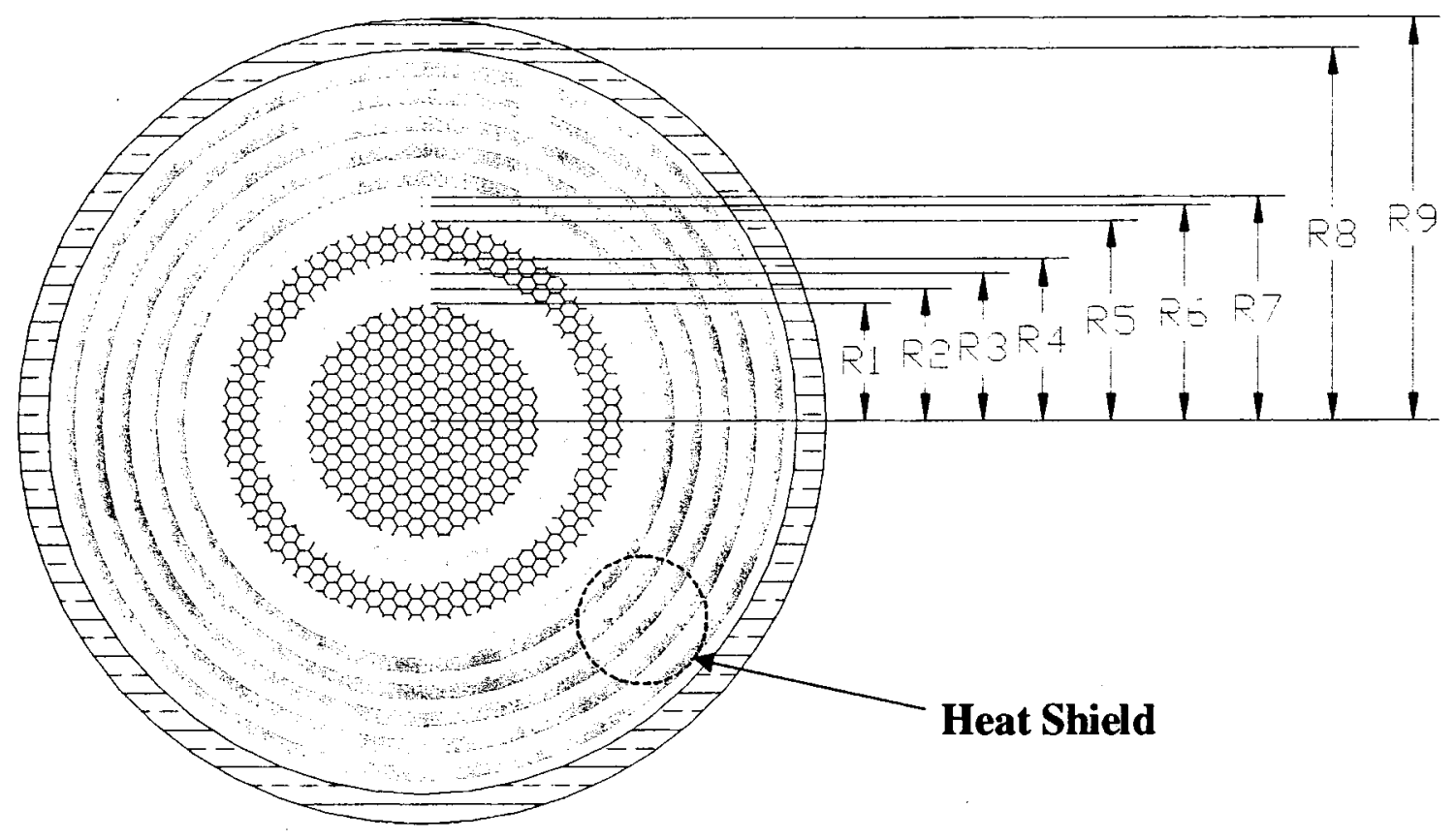

FIGURE 19. Transmission Line Cross-Section

Table 2. Transmission Line Cross-Section Dimensions

\begin{tabular}{|r|c|c|c|c|c|c|c|c|c|}
\hline Coil \# & $\begin{array}{c}R 1 \\
{[\mathrm{~cm}]}\end{array}$ & $\begin{array}{c}R 2 \\
{[\mathrm{~cm}]}\end{array}$ & $\begin{array}{c}R 3 \\
{[\mathrm{~cm}]}\end{array}$ & $\begin{array}{c}R 4 \\
{[\mathrm{~cm}]}\end{array}$ & $\begin{array}{c}R 5 \\
{[\mathrm{~cm}]}\end{array}$ & $\begin{array}{c}R 6 \\
{[\mathrm{~cm}]}\end{array}$ & $\begin{array}{c}R 7 \\
{[\mathrm{~cm}]}\end{array}$ & $\begin{array}{c}R 8 \\
{[\mathrm{~cm}]}\end{array}$ & $\begin{array}{r}R 9 \\
{[\mathrm{~cm}]}\end{array}$ \\
\hline $\mathbf{1}$ & 0.27 & 0.37 & 0.39 & 0.49 & 0.56 & 0.66 & 0.68 & 1.68 & 1.88 \\
\hline $\mathbf{2}$ & 0.30 & 0.40 & 0.43 & 0.53 & 0.61 & 0.71 & 0.73 & 1.73 & 1.93 \\
\hline $\mathbf{3}$ & 0.34 & 0.44 & 0.48 & 0.58 & 0.67 & 0.77 & 0.79 & 1.79 & 1.99 \\
\hline $\mathbf{4}$ & 0.39 & 0.49 & 0.53 & 0.63 & 0.74 & 0.84 & 0.87 & 1.87 & 2.07 \\
\hline $\mathbf{5}$ & 0.44 & 0.54 & 0.59 & 0.69 & 0.82 & 0.92 & 0.95 & 1.95 & 2.15 \\
\hline $\mathbf{6}$ & 0.52 & 0.62 & 0.68 & 0.78 & 0.93 & 1.03 & 1.07 & 2.07 & 2.27 \\
\hline $\mathbf{7}$ & 0.62 & 0.72 & 0.80 & 0.90 & 1.09 & 1.19 & 1.24 & 2.24 & 2.44 \\
\hline $\mathbf{8}$ & 0.79 & 0.89 & 0.99 & 1.09 & 1.35 & 1.45 & 1.51 & 2.51 & 2.71 \\
\hline
\end{tabular}




\section{PROPELLANT STORAGE AND SUPPLY}

The MTF device requires three separate propellants, each with its own separate storage and supply system.

A $50 \%-50 \%$ molar mixture of deuterium and tritium is stored as a high-pressure gas and, after suitable pressure regulation, fed to the two conical theta pinches to produce the spheromaks, which ultimately merge to create the FRC target plasma. The very small quantity of plasma required to create the target (approximately 1 milligram per pulse), coupled with the need for a relatively precise mixture of the two isotopes, makes common storage attractive. Statistical mechanics naturally ensures a virtually perfect $50 \%-50 \%$ molar mixture, without any need for complex independent metering systems for the two gases.

The plasma gun system requires two separate propellant supply systems. One feeds deuterium, for use at the very front of the gun's discharge plasma stream. The second, which provides the bulk of the plasma, is of (normal isotope) hydrogen. For the hydrogen, which comprises the bulk of the total propellant load, volume constraints make liquid storage the only viable option. This does not pose significant problems provided some care is taken over the thermal protection system for the tanks. As the HOPE destination is in the outer solar system, maintaining low temperatures is less of a problem than in Earth orbit. High efficiency cryocoolers can be used to balance the natural heat leakage into the hydrogen and thus maintain the liquid state. Storage of the relatively small quantity of deuterium in liquid form is actually somewhat easier than normal hydrogen, the boiling point being several degrees higher. For reference, note that the entire system of plasma guns (i.e. all 48 of them) consumes approximately 50 milligrams of deuterium and 850 milligrams of hydrogen per fusion pulse.

The plasma guns can be designed to operate using gas, liquid or even solid propellants. Given their location around the MTF nozzle, in an area of elevated temperature, gaseous feed is clearly the favored option. An overall schematic for the storage and supply system is given in Figure 20.

Note that the four deuterium-tritium tanks are each shaded slightly differently. This is to denote a slight variation in the proportions of the two gasses at loading. The tank intended for immediate use is loaded in a straight 50\%-50\% molar mixture. The tanks intended for use later in the mission are loaded slightly tritiumrich. This is to offset the natural radioactive decay of tritium (into helium-3). The later the tank's intended use, the more tritium-rich is its initial load.

Note also the use of gas compressors and plenum chambers. The compressors are needed to overcome line losses on the way down to the plasma guns. The plenum chambers are to ensure reasonably constant gun inlet conditions.

Provision is also made for the transfer of liquid hydrogen between the various storage tanks, in order that the vehicle center of mass can be controlled. 


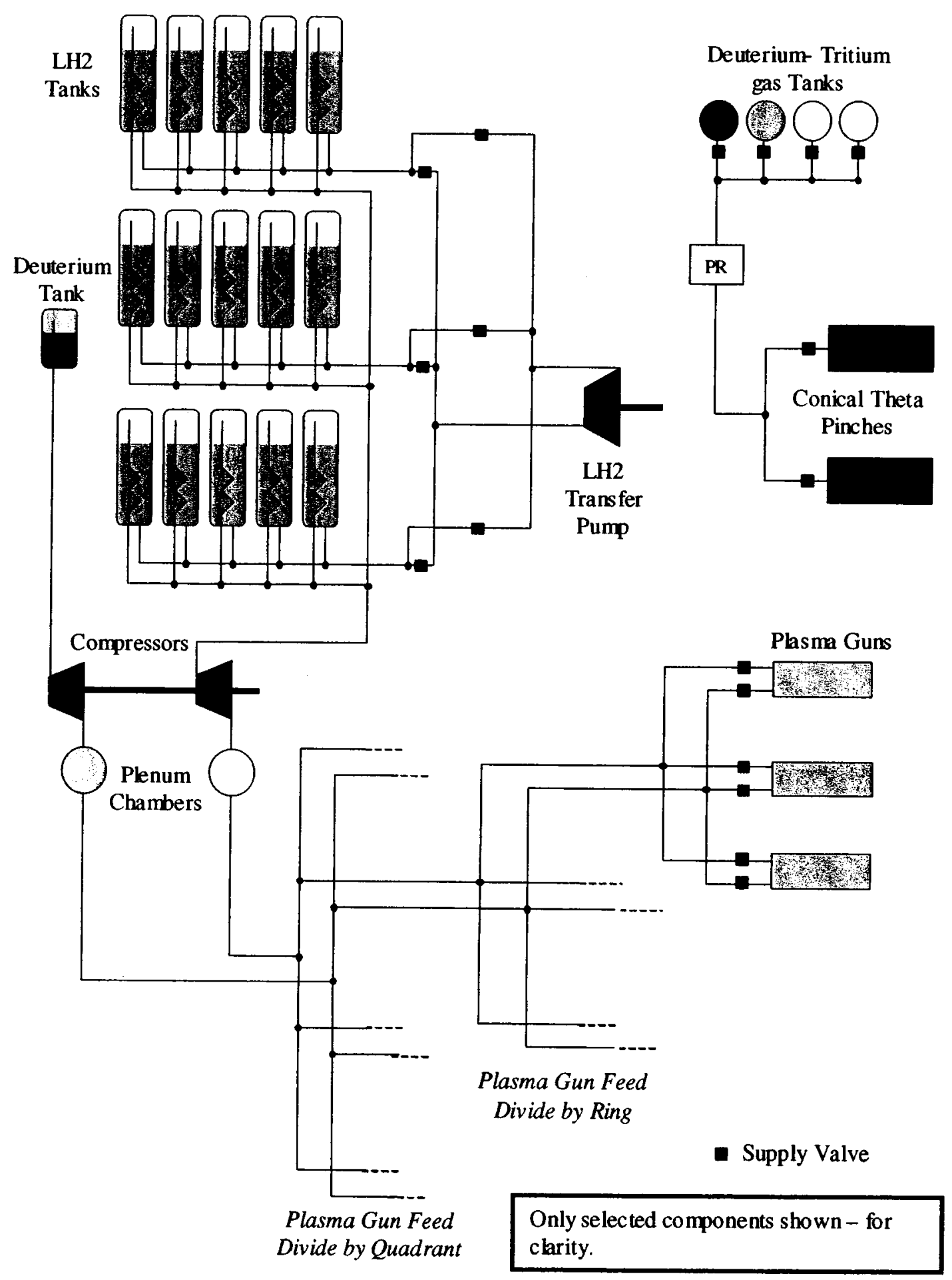

FIGURE 20. Propellant Storage and Supply System 


\section{MTF MASS ESTIMATE}

A simple mass breakdown for the MTF system, excluding the propellant storage and supply systems, is given in Table 3.

Table 3. MTF Device Mass Summary

\begin{tabular}{|c|c|c|}
\hline Component & Mass (kg) & Notes \\
\hline Plasma Guns & 2,219 & 48 plasma guns \\
\hline Capacitors & 6,657 & Provide power to the 48 plasma guns \\
\hline Target Generator System & 666 & Two conical theta pinches \\
\hline Nozzle Structure & 20,576 & Splines and rings \\
\hline Nozzle Structure Neutron Shield & 16,036 & Neutron shielding for the nozzle splines and rings. \\
\hline $\begin{array}{l}\text { Nozzle Coils and Coil Neutron } \\
\text { Shields }\end{array}$ & 33,400 & $\begin{array}{l}\text { Seed and Main Coils, together with associated } \\
\text { neutron shielding. }\end{array}$ \\
\hline $\begin{array}{l}\text { Superconducting Magnetic Energy } \\
\text { Storage (SMES) System }\end{array}$ & 3,000 & $\begin{array}{l}\text { Stores electrical energy (required to generate the } \\
\text { seed magnetic field) between pulses of the MTF } \\
\text { system. }\end{array}$ \\
\hline Re-Charging Circuit & 1,664 & $\begin{array}{l}\text { Circuit which routes power between the coils and the } \\
\text { SMES }\end{array}$ \\
\hline Vehicle Neutron Shield & 37,000 & $\begin{array}{l}\text { Water tank located above nozzle apex to shield the } \\
\text { vehicle from fusion neutrons. }\end{array}$ \\
\hline Power Cables & 115 & \\
\hline Total: & 121,333 & \\
\hline
\end{tabular}

These mass values apply to a device pulsing at a frequency of $20 \mathrm{~Hz}$ and with a mean jet power of $2.038 \mathrm{GW}$.

\section{MTF PERFORMANCE SUMMARY}

A simple mass breakdown for the MTF system, excluding the propellant storage and supply systems, is given in Table 4.

Table 4. MTF Device Performance Summary

\begin{tabular}{|l|c|}
\hline \multicolumn{1}{|c|}{ Parameter } & Nominal Value \\
\hline MTF Mean Jet Power & $2.038 \mathrm{GW}$ \\
\hline MTF Pulse Repetition Frequency & $20 \mathrm{Hertz}$ \\
\hline Specific Impulse & $70,485 \mathrm{~seconds}$ \\
\hline Mean Thrust & $5,798 \mathrm{Newton}$ \\
\hline Total mean heat input rate to MTF device due to intercepted 14.1 MeV neutrons & $317.7 \mathrm{MW}$ \\
\hline Total mean heat input rate to MTF device due to intercepted 2.45 MeV neutrons & $44.4 \mathrm{MW}$ \\
\hline Assumed waste heat rate generated by secondaries per neutron capture & $1 \mathrm{MeV}$ \\
\hline Heat transfer rate from plasma to structure (primarily Bremsstrahlung) & $85.0 \mathrm{MW}$ \\
\hline $\begin{array}{l}\text { Rate of heat generation due to nozzle components (plasma guns, target generators, } \\
\text { ohmic heating of coils) }\end{array}$ & $45.8 \mathrm{MW}$ \\
\hline
\end{tabular}




\section{DEUTERIUM HELIUM-3 MTF}

Thus far all discussion has centered upon an MTF device using a deuterium-tritium trigger to initiate fusion and an all-deuterium inner liner to provide the main fusion energy yield. There is an alternate and higher performing fusion fuel combination which should be available in the future. The trigger plasma would still consist of a $50 \%$ - 50\% (molar) deuterium-tritium plasma, but now the plasma in the inner portion of the liner would no longer be pure deuterium; instead a 50\% - 50\% molar mixture of deuterium and helium-3 would be used. Although this combination requires a higher ignition temperature than the all-deuterium option, it is still achievable with the same deuterium-tritium trigger. Once initiated, the deuterium-helium-3 fusion reaction offers both an improved level of energy release, compared to the deuterium-deuterium baseline, and also a lower neutron flux. With both an improved energy release and a larger proportion of the liberated energy appearing in the form of charged particle kinetic energy, significant performance improvements will result. Charged particle kinetic energy can be absorbed and re-directed by the nozzle magnetic field, thus adding useful impulse to the vehicle. By comparison, neutron kinetic energy places a significant mass burden on the vehicle due to the shielding requirement. Although the deuterium-helium-3 reaction does still produce some neutrons, the number will be reduced compared with the all-deuterium inner liner option.

The drawback of this advanced fuel option is the relative scarcity of helium-3. Although it can be produced by the decay of tritium, this technique is inherently limited by the scarcity of tritium, which must itself be produced by neutron bombardment within a nuclear reactor. Only trace amounts of helium-3 are available naturally on the Earth, although it is postulated that larger amounts might be available at significant depths. A plentiful supply exists in the lunar regolith, which accumulates helium-3 as a result of solar wind bombardment. Although at present this may seem a somewhat inaccessible deposit, it should be considered within the context of the cislunar infrastructure likely to be in place by the time an MTF propulsion system is in operation. By this time, with MTF providing the means for human exploration of the outer solar system, thriving transport, manufacturing and resource extraction infrastructure should be in place both at the Earth's Lagrange points and on the lunar surface. The process of helium-3 extraction from lunar regolith has already been seriously studied, with encouraging results (Wittenberg, 1992).

Even more extensive sources of helium-3 are almost certainly available in the outer solar system. All of the gas giant planets appear to contain substantial quantities of the isotope. Although the delta-vs associated with atmospheric flight within a gas giant are formidable, it is possible that "mining" operations may eventually be possible. The first MTF flights to the outer solar system might use deuterium, once appropriate infrastructure has been established, subsequent flights might be able to use helium- 3 .

\section{ACKNOWLEDGMENTS}

This work was performed under the auspices of the HOPE Study, which constitutes Group 2 of the 2002 RASC Program. The authors would like to acknowledge the guidance and assistance of the HOPE management team at the NASA Langley Research Center as well as fruitful discussions with other members of the HOPE team, particularly those at the NASA Glenn Research Center.

\section{REFERENCES}

Adams, R.B. et al Crewed Mission to Callisto Using Advanced Plasma Propulsion Systems. Paper to be presented at the Space Technology Application International Forum, 2003.

Bond, A. et al Project Daedalus Final Report. Published by the British Interplanetary Society, 1978.

Thio, Y.C.F., Freeze, B., Kirkpatrick, R.C., Landrum, B., Gerrish, H. and Schmidt, G.R., High-Energy Space

Propulsion Based on Magnetized Target Fusion, AIAA 99-2703, 1999. 
Wittenberg, L.J., Cameron, E.N., Kulcinski, G.L., Ott, S.H., Santarius, J.F., Sviatoslavsky, G.I., Sviatoslavsky, I.N. and Thompson, H.E., A Review of ${ }^{3}$ He Resources and Acquisition for Use as a Fusion Fuel. Fusion Technology, Vol. 21, July 1992. 